\title{
Comparison and combination of a hemodynamics/biomarkers-based model with simplified PESI score for prognostic stratification of acute pulmonary embolism: findings from a real world study
}

Luca Masotti $^{17 *}$, Grazia Panigada ${ }^{2}$, Giancarlo Landini ${ }^{1}$, Filippo Pieralli ${ }^{3}$, Francesco Corradi ${ }^{3}$, Salvatore Lenti ${ }^{4}$, Rino Migliacci ${ }^{5}$, Carlo Nozzoli ${ }^{3}$, Maddalena Grazzini ${ }^{3}$, Lucia Ciucciarelli ${ }^{3}$, Alessandro Morettini ${ }^{3}$, Sara Bucherelli ${ }^{3}$, Alessandra Petrioli ${ }^{3}$, Carlotta Casati ${ }^{3}$, Mario Felici ${ }^{4}$, Luciano Ralli ${ }^{4}$, Stefano Arrigucci ${ }^{4}$, Laila Teghini ${ }^{2}$, Giovanni Antonio Porciello ${ }^{5}$, Stefano Spolveri ${ }^{6}$, Daniele Baldoni ${ }^{6}$, Anna Frullini ${ }^{6}$, Barbara Cimolato ${ }^{1}$, Gianni Lorenzini ${ }^{7}$, Alessandro Pampana ${ }^{7}$, Guidantonio Rinaldi ${ }^{8}$, Maria Chiara Bertieri ${ }^{8}$, Raffaele Laureano ${ }^{9}$, Stefano Tatini ${ }^{9}$, Alberto Fortini ${ }^{10}$, Chiara Angotti ${ }^{10}$, Valerio Verdiani ${ }^{11}$,

Anna Maria Romagnoli ${ }^{11}$, Irene Cascinelli ${ }^{11}$, Alberto Camaiti ${ }^{12}$, Nicola Mumoli ${ }^{12}$, Marco Cei ${ }^{12}$, Stefano Giuntoli ${ }^{12}$, Massimo Alessandri ${ }^{13}$, Alessandro De Palma ${ }^{13}$, Maurizio Manini ${ }^{14}$, Veronica De Crescenzo ${ }^{14}$, Michele Piacentini ${ }^{15}$, Carlo Passaglia ${ }^{16}$, Giancarlo Tintori ${ }^{16}$, Carlo Palermo $^{17}$, Alba Dainelli ${ }^{17}$, Roberto Andreini ${ }^{18}$, Giuseppa Levantino ${ }^{18}$, Plinio Fabiani ${ }^{19}$, Lucia Raimondi $^{19}$, Massimo Di Natale ${ }^{20}$, Filippo Risaliti ${ }^{20}$, Rossella Nassi ${ }^{21}$, Roberta Mastriforti ${ }^{21}$, Roberto Cappelli ${ }^{22}$, Michele Voglino ${ }^{22}$, Paola Lambelet $^{23}$, Stefano Fascetti ${ }^{23}$, Adriano Cioppi ${ }^{23}$, Valentina Carli ${ }^{23}$, Alessandro Tafi ${ }^{24}$, Simone Meini ${ }^{24}$, Emilio Santoro ${ }^{25}$, Claudia Rosi ${ }^{25}$

\footnotetext{
${ }^{1}$ Medicina Interna, Ospedale Santa Maria Nuova, Firenze, Italy, ${ }^{2}$ Medicina Interna, Ospedale di Pescia, Pescia, Italy, ${ }^{3}$ Medicina Interna, Azienda Ospedaliera Universitaria Careggi, Firenze, Italy, ${ }^{4}$ Medicina Interna, Ospedale San Donato, Arezzo, Italy, ${ }^{5}$ Medicina Interna, Ospedale di Cortona, Cortona, Italy, ${ }^{6}$ Medicina Interna, Ospedale di Borgo San Lorenzo, Firenze, Italy, ${ }^{7}$ Medicina Interna, Ospedale di Cecina, Italy, ${ }^{8}$ Medicina Interna Ospedale di Barga Lucca, Italy, ${ }^{9}$ Medicina Interna, Ospedale Santa Maria Annunziata, Firenze, Italy, ${ }^{10}$ Medicina Interna, Ospedale San Giovanni di Dio, Firenze, Italy, ${ }^{11}$ Medicina Interna Ospedale di Grosseto, Italy, ${ }^{12}$ Medicina Interna Ospedale di Livorno, Italy, ${ }^{13}$ Medicina Interna, Ospedale di Massa Marittima, Grosseto, Italy, ${ }^{14}$ Medicina Interna, Ospedale di Orbetello Grosseto, Italy, ${ }^{15}$ Medicina Interna Ospedale di Piombino, Italy, ${ }^{16}$ Medicina Interna, Ospedale Cisanello Pisa, Italy, ${ }^{17}$ Medicina Interna Ospedale di Campostaggia Siena, Italy, ${ }^{18}$ Medicina Interna Ospedale di Pontedera, Italy, ${ }^{19}$ Medicina Interna Ospedale di Portoferraio Livorno, Italy, ${ }^{20}$ Medicina Interna Ospedale di Prato, Italy, ${ }^{21}$ Medicina Interna Ospedale di San Sepolcro Arezzo, Italy, ${ }^{22}$ Medicina Interna Ospedale Le Scotte Siena, Italy, ${ }^{23}$ Medicina Interna Ospedale di Viareggio, Italy, ${ }^{24}$ Medicina Interna Ospedale di Volterra, Italy, ${ }^{25}$ Medicina Interna Ospedale di Bibbiena, Arezzo, Italy
}

Received: 11 September 2015

Accepted: 07 October 2015

*Correspondence:

Dr. Luca Masotti,

E-mail: luca.masotti@tin.it

Copyright: (C) the author(s), publisher and licensee Medip Academy. This is an open-access article distributed under the terms of the Creative Commons Attribution Non-Commercial License, which permits unrestricted non-commercial use, distribution, and reproduction in any medium, provided the original work is properly cited. 


\section{ABSTRACT}

Background: Prognostic stratification is of utmost importance for management of acute Pulmonary Embolism (PE) in clinical practice. Many prognostic models have been proposed, but which is the best prognosticator in real life remains unclear. The aim of our study was to compare and combine the predictive values of the hemodynamics/biomarkers based prognostic model proposed by European Society of Cardiology (ESC) in 2008 and simplified PESI score (sPESI).

Methods: Data records of 452 patients discharged for acute PE from Internal Medicine wards of Tuscany (Italy) were analysed. The ESC model and sPESI were retrospectively calculated and compared by using Areas under Receiver Operating Characteristics (ROC) Curves (AUCs) and finally the combination of the two models was tested in hemodinamically stable patients. All cause and PE-related in-hospital mortality and fatal or major bleedings were the analyzed endpoints

Results: All cause in-hospital mortality was 25\% (16.6\% PE related) in high risk, $8.7 \%(4.7 \%)$ in intermediate risk and $3.8 \%(1.2 \%)$ in low risk patients according to ESC model. All cause in-hospital mortality was $10.95 \%(5.75 \%$ PE related) in patients with sPESI score $\geq 1$ and $0 \%(0 \%)$ in sPESI score 0 . Predictive performance of sPESI was not significantly different compared with 2008 ESC model both for all cause (AUC sPESI 0.711, 95\% CI: 0.661-0.758 versus ESC 0.619, 95\% CI: 0.567-0.670, difference between AUCs 0.0916, p=0.084) and for PE-related mortality (AUC sPESI 0.764, 95\% CI: 0.717-0.808 versus ESC 0.650, 95\% CI: 0.598-0.700, difference between AUCs 0.114 , $\mathrm{p}=0.11$ ). Fatal or major bleedings occurred in $4.30 \%$ of high risk, $1.60 \%$ of intermediate risk and $2.50 \%$ of low risk patients according to 2008 ESC model, whereas these occurred in $1.80 \%$ of high risk and $1.45 \%$ of low risk patients according to sPESI, respectively. Predictive performance for fatal or major bleeding between two models was not significantly different (AUC sPESI 0.658, 95\% CI: 0.606-0.707 versus ESC 0.512, 95\% CI: 0.459-0.565, difference between AUCs 0.145, p=0.34). In hemodynamically stable patients, the combined endpoint in-hospital PE-related mortality and/or fatal or major bleeding (adverse events) occurred in $0 \%$ of patients with low risk ESC model and sPESI score 0 , whilst it occurred in $5.5 \%$ of patients with low-risk ESC model but sPESI $\geq 1$. In intermediate risk patients according to ESC model, adverse events occurred in $3.6 \%$ of patients with sPESI score 0 and $6.65 \%$ of patients with sPESI score $\geq 1$.

Conclusions: In real world, predictive performance of sPESI and the hemodynamic/biomarkers-based ESC model as prognosticator of in-hospital mortality and bleedings is similar. Combination of sPESI 0 with low risk ESC model may identify patients with very low risk of adverse events and candidate for early hospital discharge or home treatment.

Keywords: Pulmonary embolism, Prognosis, Biomarkers; PESI, Echocardiography, Bleeding, ESC

\section{INTRODUCTION}

Acute Pulmonary Embolism (PE) is a major cardiovascular emergency, burdened for high mortality, especially when associated with hemodynamic instability. ${ }^{1}$

Prognostic stratification of acute PE is of utmost importance for the choice of appropriate treatment and setting of care. It can be assured by using clinical, instrumental and laboratory assessment. ${ }^{2,3}$

Many prognostic models for risk stratification of acute PE have been proposed, but which is the best prognosticator remains unclear, especially in daily real practice. $^{4}$

In 2008 for the first time, the European Society of Cardiology proposed a model based on hemodynamics and cardiac biomarkers. ${ }^{5}$ This model has represented a cornerstone in acute management of PE. In fact, for the first time, recommendations on acute PE suggested to manage it on early mortality risk. Briefly, the 2008 ESC model identified high risk PE when patients presented with shock or hypotension, intermediate risk PE when patients, despite normotensive, presented echocardiographic pattern and/or cardiac biomarkers of Right Heart Dysfunction (RHD) or myocardial damage such as natriuretic peptides or troponins and finally low risk PE when patients were normotensive and without echocardiographic and biomarker signs of RHD or myocardial damage. ${ }^{5}$ According to 2008 ESC model, early mortality risk was $>15 \%$ in high risk, $3-15 \%$ in intermediate risk and $\leq 1 \%$ in low risk patients. Due to the abovementioned early mortality risk, high risk PE needs for prompt reperfusion by using systemic thrombolysis or embolectomy and closer monitoring in Intensive Care Units, intermediate risk PE needs for close monitoring due the risk of hemodynamic deterioration and low risk $\mathrm{PE}$ does not require close monitoring and may be managed with short hospital stay or at home treatment. Haemodinamically stable PE require treatment by using low molecular weight or unfractioned heparins or fondaparinux overlapped by vitamin $\mathrm{K}$ antagonists or, 
much recently, by using a single drug approach with direct oral anticoagulants (DOACs). ${ }^{5,6}$ The 2008 ESC model was proposed without validation studies but based on Experts opinion made on literature evidence. Latter, the prognostic power of 2008 ESC model has been validated. $^{7}$

Despite hemodynamic aspects are of utmost importance for prognostic stratification of acute PE, many other risk factors for early mortality have been demonstrated. ${ }^{8} \mathrm{Co}-$ morbidity seems to influence mortality as well as hemodynamic status. Therefore, one of the main limitation of the 2008 ESC prognostic model is the lack of prognostic burden derived from co-morbidity such age, cardiopulmonary diseases and cancer.

Pulmonary Embolism Severity Index (PESI) is a prognostic model which considers many co-morbid conditions. ${ }^{9}$ Firstly, it was validated in its original version in 2007 by Aujesky D et al. ${ }^{9}$ It considers eleven variables [age, male sex, cancer, heart failure, Chronic Obstructive Pulmonary Diseases (COPD), Heart Rate (HR), Systolic Blood Pressure (SBP), respiratory rate, body temperature, mental status alteration, oxygen arterial saturation], combination of them leading to five risk classes for 30 days mortality. 30-days mortality in classes I and II (low risk) is $0.7 \%$ and $1.2 \%$ respectively, whereas 30 -days mortality in classes III, IV and V (high risk) is $4.8 \%$, $13.6 \%$ and $25 \%$ respectively. ${ }^{9}$ Latter, a simplified version of PESI (sPESI) score was validated in 2010 by Jimenez $\mathrm{D}$ et al. ${ }^{10}$ The simplified version of PESI considers the presence or absence of age over 80 years, history of cancer, heart failure or COPD, HR $\geq 110$ beats for minute, $\mathrm{SBP} \leq 100 \mathrm{mmHg}$, oxygen arterial saturation $\leq 90 \%$. The contemporary absence of all variables (sPESI score 0) identify patients at $\leq 1 \%$ risk for 30 -days mortality, named low risk, whereas the presence of at least one of variables (sPESI score $\geq 1$ ) identifies patients at high risk for 30 days mortality, being $10.9 \%$ in the validation study. ${ }^{10}$ Both version of PESI score, original and simplified, have been used as prognostic model for identifying low risk patients candidate for home treatment. ${ }^{11-13}$

Despite this background, literature evidence reporting on which is the best prognosticator for adverse outcome in patients suffering for acute PE in real world lacks. Therefore, the aim of the present study was to compare and combine the predictive values of 2008 European Society of Cardiology prognostic model (ESC) and simplified PESI score (sPESI) as prognosticator for inhospital mortality and fatal and/or major bleedings.

\section{METHODS}

We performed a multicenter, observational, retrospective, cohort study aimed to evaluate characteristics and clinical management of acute PE patients admitted in Internal Medicine wards of Tuscany. Demographic, clinical and prognostic data of at least ten patients consecutively discharged between 2012 and 2013 years from each
Center for acute PE were provided. The 2008 ESC prognostic model and sPESI were retrospectively calculated and compared. Echocardiographic RHD was defined in the presence of four chambers end-diastolic $\mathrm{RV}$ diameter/LV diameters ratio $\geq 1$. Biomarker signs of RHD and/or myocardial injury were considered positive if brain natriuretic pepited (BNP) was higher than 300 $\mathrm{pg} / \mathrm{mL}$ or its terminal portion (NT-proBNP) was higher than $900 \mathrm{ng} / \mathrm{L}$ and troponin I was higher than upper limit of normality cut-off in single Center. Accordingly, in hemodynamically stable patients, sPESI score and 2008 ESC prognostic model were combined with the aim to obtain four classes: A) low risk ESC model plus sPESI 0, B) low risk ESC model plus $\mathrm{sPESI} \geq 1, \mathrm{C}$ ) intermediate risk ESC model plus sPESI 0 and D) intermediate risk ESC model plus sPESI $\geq 1$. All-cause in-hospital mortality, PE-related mortality and fatal and/or major bleedings according to the ISTH definition were the observed endpoints. ${ }^{14}$ PE-related mortality was defined as mortality directly due to $\mathrm{PE}$ and its hemodynamic consequences. The combination of PE-related mortality and fatal of major bleedings were defined as combined adverse events.

Variables were reported as means \pm Standard Deviation (SD). For statistical analysis, categorical variables were compared by using the Fisher's exact test, whereas continuous data were compared by using t test of Student. Area under Receiver Operating Characteristic (ROC) curves (AUCs) were constructed for each study endpoint in order to examine the predictive performance of each prognostic model. AUCs were compared using the nonparametric method. Significance was indicated by a $p$ value $<0.05$.

\section{RESULTS}

452 patients were enclosed in the study. Table 1 shows the general characteristics of patients.

Echocardiography was performed in $70.1 \%$ of patients, BNP or NT-proBNP assay in $35.6 \%$ and troponin I assay in $80.7 \%$. sPESI score was calculated in $100 \%$ of patients, whereas we are able to apply the ESC prognostic model in 354 patients $(78.3 \%)$ on whom, finally, we performed the comparison between the two models.

Overall, in-hospital mortality was $25 \%$ (16.6\% PE related) in high risk, $8.7 \%(4.7 \%)$ in intermediate risk and $3.8 \%(1.2 \%)$ in low risk ESC model (Figure 1). Inhospital mortality was $10.95 \%(5.75 \%$ PE related) in patients with sPESI score $\geq 1$ and $0 \%(0 \%)$ in sPESI score 0 . Significance was found between mortality in high risk and intermediate risk patients $(p=0.019$ for all-cause mortality, $\mathrm{p}=0.034$ for PE-related mortality) and between mortality in high risk and low risk patients $(\mathrm{p}=0.0038$ for all-cause mortality, $\mathrm{p}=0.0088$ for PE-related mortality), whereas no significance was found between mortality in intermediate risk and low risk patients $(\mathrm{p}=0.22$ for allcause mortality and $\mathrm{p}=0.20$ for PE-related mortality). 
Mortality increased from $0 \%$ in patients with sPESI score 0 to $29.1 \%$ (20.8\% PE related) in patients with sPESI score $\geq 4$ (Figure 2).

Predictive power for all cause in-hospital mortality of sPESI score 2008 ESC prognostic model was not statistically different (AUC sPESI 0.711, 95\% CI: 0.6610.758 versus ESC 0.619, 95\% CI: 0.567-0.670, difference between AUCs 0.0916, $\mathrm{p}=0.084$ ) as well as for PErelated mortality (AUC sPESI 0.764, 95\% CI: 0.717 0.808 versus ESC $0.650,95 \%$ CI: 0.598-0.700, difference between AUCs 0.114, p=0.11) (Figure 3).

Fatal or major bleedings occurred in $4.30 \%$ of high risk, $1.60 \%$ of intermediate risk and $2.50 \%$ of low risk patients according to ESC model (Figure 4), whereas these occurred in $1.80 \%$ of high risk and $1.45 \%$ of low risk patients according to sPESI score (Figure 5). Significance was not found in bleeding rate between risk subgroups both for ESC model and sPESI score.

Table 1: General characteristics of patients.

\begin{tabular}{|c|c|}
\hline \multicolumn{2}{|l|}{ General characteristics of patients } \\
\hline Number & 452 \\
\hline Males & $39.8 \%$ \\
\hline Females & $60.2 \%$ \\
\hline Mean age $($ years $) \pm S D$ & $76.01 \pm 12.34$ \\
\hline Mean length of hospital stay (days) \pm SD & $10.60 \pm 6.63$ \\
\hline \multicolumn{2}{|l|}{ In-hospital mortality } \\
\hline All-cause & $9.5 \%$ \\
\hline PE related & $5.7 \%$ \\
\hline \multicolumn{2}{|l|}{2008 ESC prognostic model distribution } \\
\hline High risk & $5.1 \%$ \\
\hline Intermediate risk & $55.8 \%$ \\
\hline Low risk & $17.5 \%$ \\
\hline Not applicable & $21.6 \%$ \\
\hline \multicolumn{2}{|l|}{ sPESI score distribution } \\
\hline Low risk & $15.2 \%$ \\
\hline High risk & $84.8 \%$ \\
\hline 1 & $39.2 \%$ \\
\hline 2 & $27.7 \%$ \\
\hline 3 & $12.6 \%$ \\
\hline$\geq 4$ & $5.3 \%$ \\
\hline \multicolumn{2}{|l|}{ sPESI variables distribution } \\
\hline Age $\geq 80$ years & $45.3 \%$ \\
\hline Active cancer & $30.2 \%$ \\
\hline Heart failure or COPD & $22.4 \%$ \\
\hline Heart rate $\geq 110$ beats for minute & $19.8 \%$ \\
\hline Systolic blood pressure $\leq 100 \mathrm{mmHg}$ & $7.3 \%$ \\
\hline Oxygen arterial saturation $\leq 90 \%$ & $27.4 \%$ \\
\hline \multicolumn{2}{|c|}{ In-hospital bleedings according to ISTH criteria } \\
\hline Overall & $3.1 \%$ \\
\hline Fatal bleedings & $0.2 \%$ \\
\hline Non-fatal major bleedings & $1.5 \%$ \\
\hline Non major bleedings & $1.4 \%$ \\
\hline
\end{tabular}

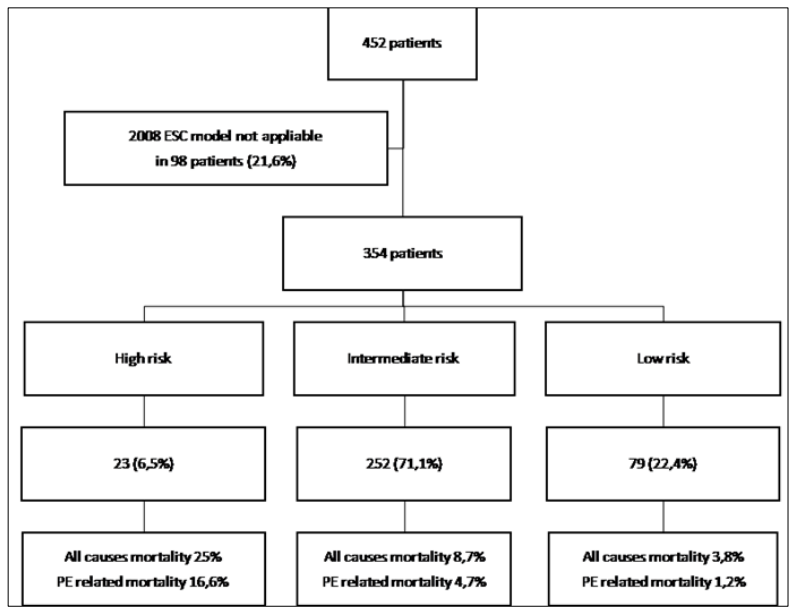

Figure 1: 2008 ESC prognostic model and in-hospital mortality.

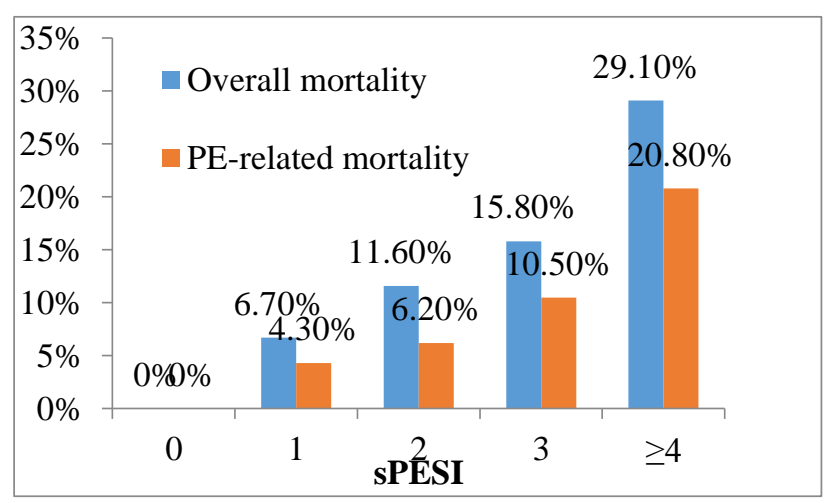

Figure 2: sPESI score and in-hospital mortality.

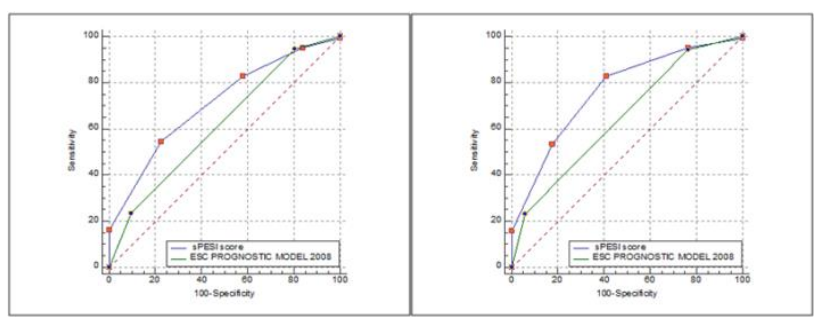

Figure 3: ROC curves for predictive value for inhospital mortality, overall and PE-related, of 2008 ESC prognostic model and sPESI score. All-cause mortality (left), PE-related mortality (right).

Predictive value for fatal or major bleeding between two models was not significantly different (AUC sPESI 0.658, 95\% CI: $0.606-0.707$ versus ESC 0.512, 95\% CI: 0.459-0.565, difference between AUCs 0.145, $\mathrm{p}=0.34$ ) (Figure 6).

In hemodynamically stable patients, adverse events occurred in $0 \%$ of sub-group A patients (low risk ESC model combined with sPESI score 0), whilst these occurred in $5.5 \%$ of sub-group B patients (low-risk 2008 ESC model and $\mathrm{sPESI} \geq 1)(\mathrm{p}<0.01)$. In intermediate risk patients according to 2008 ESC model, adverse events 
occurred in $3.6 \%$ of sub-group $\mathrm{C}$ patients (combined with sPESI score 0 ) and $6.65 \%$ of sub-group D patients (combined with sPESI score $\geq 1)(\mathrm{p}=\mathrm{ns})$ (Figure 7).

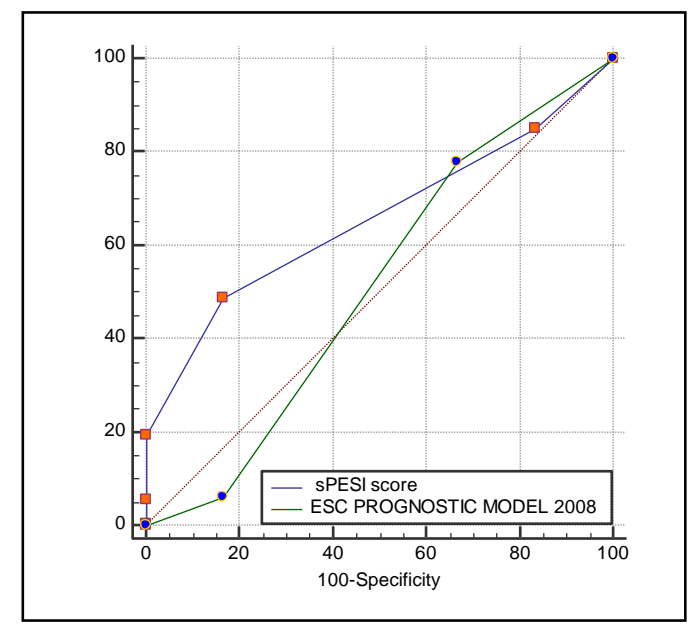

Figure 4: ROC curve for predictive value for fatal or major bleedings of 2008 ESC prognostic model and sPESI score.

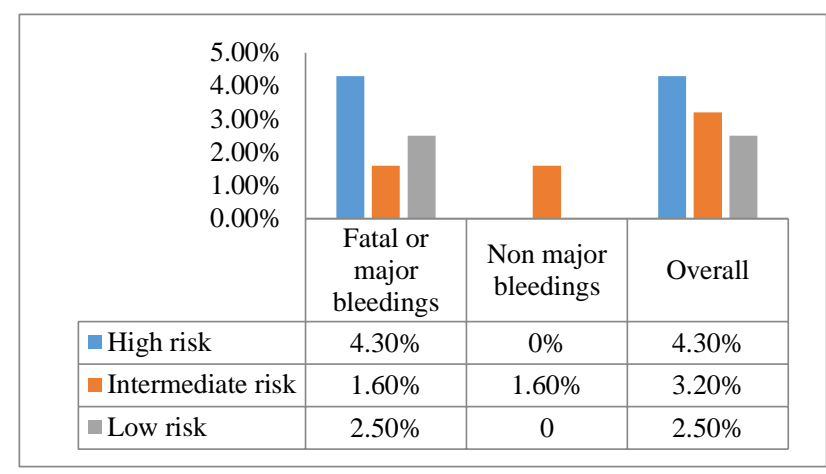

Figure 5: 2008 ESC prognostic model and bleedings.

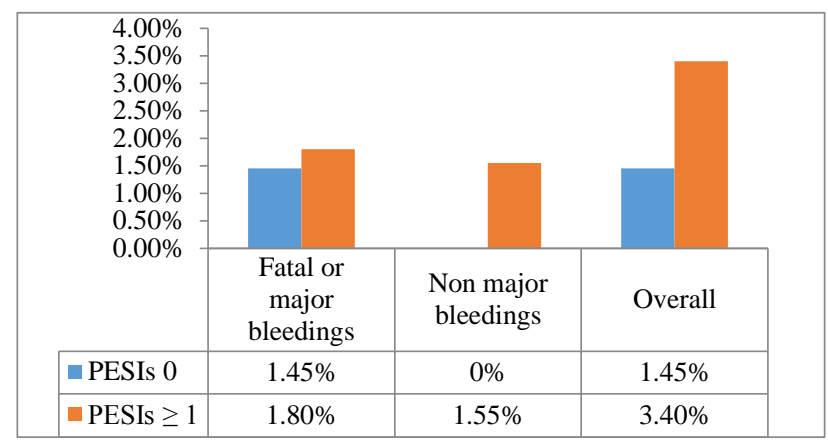

Figure 6: sPESI score and bleedings.

\section{DISCUSSION}

Management of acute PE remains a challenge in clinical practice. Prognostic stratification has become a fundamental tool for choosing appropriate treatment and setting of care. ${ }^{15}$ In the latest years prognostic scores have been derived and validated. Theoretically, an ideal prognostic score should take in account both the early mortality risk as well as bleeding risk. This is of utmost importance especially for patients candidate for early discharge or home treatment. In fact, early hospital discharge or home treatment have become a real option for PE management over the years accordingly to identification of early mortality or venous thromboembolism recurrence low risk patients. Much recently, a meta-analysis of studies on home treatment of low risk PE, the majority of them classifying low risk patients by using original or sPESI score, has demonstrated that 14-days and 90-days overall mortality, VTE recurrence and major bleedings are $0.41 \%, 0.28 \%$, $0.46 \%$ and $1.58 \%, 1.47 \%, 0.81 \%$ respectively. ${ }^{16}$

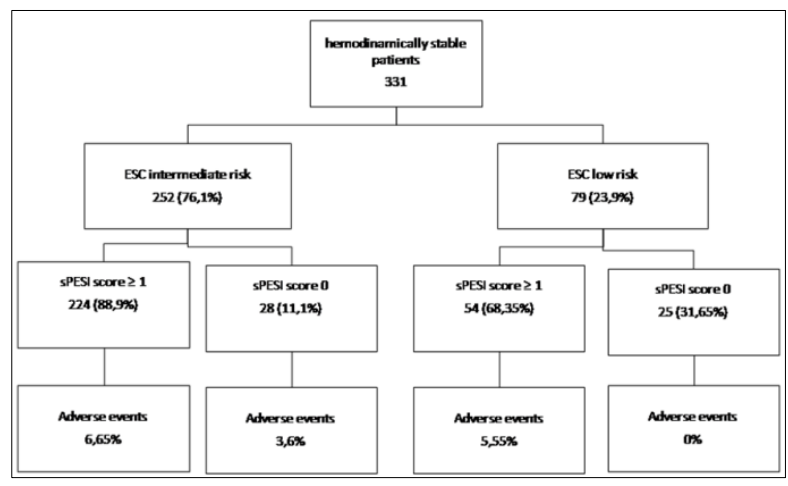

Figure 7: Combination of 2008 ESC prognostic model and SPESI score and adverse events (PE-related mortality and fatal or non-fatal major bleedings) in hemodinamically stable patients.

Despite many prognostic model have been proposed, such as the ESC prognostic model, the original and sPESI score, the Geneva prognostic model, the LR-PED, the HESTIA criteria, the Spanish score and other, which is the best prognosticator for the identification of 30-days mortality in PE patients in real world remains unclear. ${ }^{4,5,9,10,17-20}$ Moreover, the majority of the proposed prognostic models focus only on early mortality risk without bleeding risk stratification. On the other hand, few bleeding risk scores have been proposed, but, anyway, these do not give information about PE-related early mortality risk. The RIETE bleeding score has been proposed as a tool for detecting bleeding risk patients treated by using anticoagulants for venous thromboembolism. ${ }^{21}$ It takes in account variables such as age $>75$ years, metastaic cancer, bed-rest over four days, recent major bleeding, abnormal prothrombin time, severe renal failure, thrombocytopenia, anemia and distal vein thrombosis. Low risk patients according to RIETE bleeding score were found to have $0.1 \%$ of fatal bleeding during the first three months from the starting of anticoagulant therapy. However it has not been validated during hospitalization. 
A meta-analysis of studies on prognostic power of original and sPESI score enclosing twenty-five studies showed that patients with low risk PESI score have significantly less adverse outcome (combined endpoints non-fatal venous thromboembolism recurrence, non-fatal bleeding and delayed hemodynamic deterioration) compared with high risk PESI score. ${ }^{22}$ In low risk PESI score patients adverse events occurred in 1.0-2.9\% of patients compared to $2.8-39.7 \%$ in high risk PESI score. However data on adverse outcome were reported only in nine of twenty-five studies. ${ }^{22}$ Literature data reporting on bleeding events according to 2008 prognostic model lack.

Our real world study demonstrates that sPESI score, a simple and easily performable score, mainly based on history of patients and vital signs assessment, predicts allcause mortality and PE-related mortality as well as a model based on hemodynamics and biomarkers such as 2008 ESC prognostic model. However, it should be remarked that in our study all cause and $\mathrm{PE}$ related mortality was $0 \%$ in sPESI low risk compared to $3.8 \%$ and $1.2 \%$ in 2008 ESC prognostic model low risk, respectively, arising the speculation that a higher sample size of patients may lead to a statistical significance between the two scores. The good prognostic performance of sPESI is not completely surprising, since sPESI score considers both co-morbidity and hemodynamic aspects. In fact sPESI score encloses HR and SBP, the ratio of them being known as the shock index, which is a measure of hemodynamic status and has a high predictive power for poor outcome when $\geq 1 .^{23}$ Moreover sPESI score, by arterial oxygen saturation measurement, offer information about respiratory burden proved by emboli. Our study confirms findings from Lankeit $\mathrm{M}$ et al. who found no 30-day mortality in low risk sPESI score PE patients compared to $3.4 \%$ in 2008 ESC prognostic model low risk patients in a prospective study enrolling 526 patients with confirmed PE. ${ }^{24}$ Much recently Oszu S et al. reported that sPESI and 2008 ESC prognostic model showed similar performance on 30days mortality. ${ }^{25}$ They found an AUC of 0.64 (95\%CI: $0.58-0.68$ ) for 2008 ESC prognostic model and 0.62 (95\%CI: 0.57-0.67) respectively. In this study all-cause mortality in sPESI score low risk patients was $6 \%$ versus $7 \%$ in 2008 ESC prognostic model low risk. Conversely, Vanni S et al. found that 2008 ESC prognostic model predicted better than original PESI score in identifying high and low risk patients. ${ }^{26}$ In this study in-hospital allcause mortality and PE-related mortality was $3 \%$ and $1 \%$ in original PESI score low risk patients and $2 \%$ and $1 \%$ in 2008 ESC prognostic model low risk patients, respectively. $^{26}$

Few studies have analyzed the prognostic value of combination of hemodynamic parameters with comorbidity. In our study the combination of sPESI score with 2008 ESC prognostic model seems to identify the true low risk patients for adverse outcome (PE mortality and fatal/major bleedings) represented by patients at 2008 ESC low risk combined with sPESI score 0. At our knowledge this is the first literature evidence combining strategies based on hemodynamics and co-morbidity aimed to predict all adverse outcome, both mortality and bleedings. As abovementioned, this is of utmost importance, especially for making decision on early hospital discharge or home treatment, reducing the fear of physicians and increasing safety of patients. In fact, in selecting patients who could be are candidate for home treatment, safety should be taken in account as well as PE-related mortality. In our present study, patients at low risk according to 2008 ESC model presented 0\% of adverse events only when co-morbidity measured by sPESI score was absent. On the other hand patients with sPESI score 0 were at true low risk of adverse events only when hemodynamic status and biomarkers were normal since adverse events occurred in $3.6 \%$ of patients with sPESI score 0 and intermediate risk according to 2008 ESC model.

In the abovementioned study of Oszu $\mathrm{S}$ et al. the combination of sPESI score and 2088 ESC model predicted better 30-days mortality than models alone (AUC 0.69; 5\% CI: $(0.64-0.74) .^{25}$ In this study event rate of combined endpoints all cause 30-days mortality, nonfatal recurrent PE and non-fatal major bleedings was $0 \%$ when low risk patients according to ESC model were combined with sPESI $0 .^{25}$

Recently, Jimenez D et al. derived and validated a multimarker prognostic model based on sPESI as first approach. $^{27}$ They found that in patients with sPESI 0 , negative BNP identified patients with $0.9 \%$ cumulative incidence of 30-days all-cause mortality, hemodynamic deterioration and VTE recurrence, whereas positive BNP identified patients with $3.6 \%$ cumulative incidence of the abovementioned endpoints. Endpoints cumulative incidence increased from $6.1 \%$ in patients with sPESI score $\geq 1$ and negative BNP to $20.8 \%$ in patients with contemporary presence of sPESI score $\geq 1$, positive BNP, positive troponin I and proximal deep vein thrombosis. ${ }^{27}$ However, in the study of Jimenez et al. bleeding risk was not reported.

Much recently, the combination of PESI score and hemodynamic status has been proposed in the new 2014 ESC guidelines for the prognostic stratification of acute $\mathrm{PE}^{28}$ In the new ESC guidelines low risk PESI score, such as classes I-II of the original version or score 0 in the simplified version, identifies low risk PE. In patients with class I-II original PESI score or sPESI score 0, further prognostic investigations aimed to define the low mortality risk, such as trans-thoracic echocardiography and/or cardiac biomarkers are considered not necessary for this purpose. On the other side, patients with sPESI classes III-V original PESI or score $\geq 1$ require further prognostic assessment aimed to identify RHD and/or myocardial damage signs and better stratify in intermediate-high and intermediate-low risk. High risk PE definition remains unchanged and based on shock or hemodynamic instability at presentation. ${ }^{28}$ It should be 
remarked that a note of ESC suggest to enclose in intermediate risk those patients in class I-II original PESI score or sPESI score 0 who are detected to have RHD or myocardial damage signs during the diagnostic approach. $^{28}$ The new ESC model derives from Experts opinion and lacks of a clinical validation. Our findings support the importance of detecting RHD and/or myocardial damage signs making these necessary and not optional for risk stratification, especially for identification of patients with very low early mortality and bleeding risk.

\section{Strengths and limitations}

Our study analyzes data of non-selected patients admitted in more than $80 \%$ of Internal Medicine wards of Tuscany, one of the most populated regions of Italy. It offers a true real world perspective. However, we recognize that our study presents limitations, main of these being represented by the retrospective methodology. Moreover our study focus only on events occurred during hospital stay and lacks of a follow-up after it.

\section{CONCLUSION}

Prognostic stratification is a key point in the modern management of acute PE. Combination of sPESI score with a hemodynamics/biomarkers-based prognostic model such as the 2008 ESC model may stratify better the very low risk category of patient who are candidate for early hospital discharge or home treatment. This category encompass patients without hemodynamic compromise, without echocardiographic and/or biomarkers findings of RHD and/or myocardial damage and without co-morbidity such as age over 80 years and history of cardiopulmonary diseases and cancer. Prospective studies aimed to confirm our results are warranted.

\section{Funding: No funding sources}

Conflict of interest: None declared

Ethical approval: Not required

\section{REFERENCES}

1. Wood KE. Major pulmonary embolism. Review of a pathophysiologic approach to the golden hour of hemodinamically significant pulmonary embolism. Chest. 2002;121:877-905.

2. Masotti L, Righini M, Vuilleumier N, Antonelli F, Landini G, Cappelli R, et al. Prognostic stratification of acute pulmonary embolism: focus on clinical aspects, imaging, and biomarkers. Vasc Health Risk Manag. 2009;5:567-75.

3. Becattini C, Agnelli G. Acute pulmonary embolism: risk stratification in the emergency department. Intern Emerg Med. 2007;2:119-29.
4. Barra SN, Paiva L, Providência R, Fernandes A, Marques AL. A review on state-of-the-art data regarding safe early discharge following admission for pulmonary embolism: what do we know? Clin Cardio. 2013;36::507-15.

5. Torbicki A, Perrier A, Konstantinides S, Agnelli G, Galiè N, Pruszczyk $P$, et al. Guidelines on the diagnosis and management of acute pulmonary embolism: the Task Force for the Diagnosis and Management of Acute Pulmonary Embolism of the European Society of Cardiology (ESC). Eur Heart J. 2008;29:2276-315.

6. Masotti L, Lorenzini G, Landini GC, Bettoni N, Panigada G, Cappelli R. New oral anticoagulants for acute and long-term treatment of haemodynamically stable pulmonary embolism. Glob J Respir Care. 2014;1:1-8.

7. Becattini C, Casazza F, Forgione C, Porro F, Fadin BM, Stucchi A, et al. Acute pulmonary embolism: external validation of an integrated risk stratification model. Chest. 2013;144:1539-45.

8. Aujesky D, Hughes R, Jiménez D. Short-term prognosis of pulmonary embolism. J Thromb Haemos. 2009; 7(Suppl 1):318-21.

9. Aujesky D, Perrier A, Roy PM, Stone RA, Cornuz $\mathrm{J}$, Meyer $\mathrm{G}$, et al. Validation of a clinical prognostic model to identify low-risk patients with pulmonary embolism. J Intern Med. 2007;261:597-604.

10. Jiménez D, Aujesky D, Moores L, Gómez V, Lobo JL, Uresandi F, et al. Simplification of the pulmonary embolism severity index for prognostication in patients with acute symptomatic pulmonary embolism. Arch Intern Med. 2010;170:1383-9.

11. Lankeit M, Konstantinides S. Is it time for home treatment of pulmonaryembolism? Eur Respir J. 2012 Sep;40(3):742-9.

12. Squizzato A, Galli M, Dentali F, Ageno W. Outpatient treatment and early discharge of symptomatic pulmonary embolism: a systematic review. Eur Respir J. 2009;33:1148-55.

13. Aujesky D, Roy PM, Verschuren F, Righini M, Osterwalder J, Egloff M, et al. Outpatient versus inpatient treatment for patients with acute pulmonary embolism: an international, open-label, randomised, non-inferiority trial. Lancet. 2011 Jul;378(9785):41-8.

14. Schulman S, Kearon C; The Sub Committee on Control of the Anticoagulation of the Scientific and Standardization Committee of the International Society of Thrombosis and Haemostasis. Definition of major bleeding in clinical investigations of antihemostatic medicinal products in non-surgical patients. J Thromb Haemost. 2005;3:692-4.

15. Masotti L, Mannucci A, Antonelli F, Maurini V, Testa R, Marchetti S, et al. The risk-based treatment of acute pulmonary embolism. J Clin Med Res. 2009;1:1-7.

16. Piran S, Le Gal G, Wells PS, Gandara E, Righini M, Rodger MA, Carrier M. Outpatient treatment of 
symptomatic pulmonary embolism: a systematic review and meta-analysis. Thromb Res. 2013;132:515-9.

17. Barra S, Paiva L, Providência R, Fernandes A, Nascimento J, Marques AL. LR-PED rule: low risk pulmonary embolism decision rule - a new decision score for low risk pulmonary embolism. Thromb Res. 2012;130:327-33.

18. Zondag W, Hiddinga BI, Crobach MJ, Labots G, Dolsma A, Durian M, et al. Hestia criteria can discriminate high- from low-risk patients with pulmonary embolism. Eur Respir J. 2013;41:588-92.

19. Jiménez D, Yusen RD. Prognostic models for selecting patients with acute pulmonary embolism for initial outpatient therapy. Curr Opin Pulm Med. 2008 Sep;14(5):414-21.

20. Uresandi F, Otero R, Cayuela A, Cabezudo MA, Jiménez D, Laserna E, et al. A clinical prediction rule for identifying short-term risk of adverse events in patients with pulmonary thromboembolism. Arch Bronconeumol. 2007;43:617-22.

21. Nieto JA, Solano R, Trapero Iglesias N, RuizGiménez N, Fernández-Capitán C, Valero B, et al. Validation of a score for predicting fatal bleeding in patients receiving anticoagulation for venous thromboembolism. Thromb Res. 2013 Aug;132(2):175-9.

22. Zhou XY, Ben SQ, Chen HL, Ni SS. The prognostic value of pulmonary embolism severity index in acute pulmonary embolism: a meta-analysis. Respir Res. 2012;13:111.

23. Sam A, Sánchez D, Gómez V, Wagner C, Kopecna $\mathrm{D}$, Zamarro C, et al. The shock index and the simplified PESI for identification of low-risk patients with acute pulmonary embolism. Eur Respir J. 2011;37:762-6.

24. Lankeit M, Gómez V, Wagner C, Aujesky D, Recio $\mathrm{M}$, Briongos $\mathrm{S}$, et al. A strategy combining imaging and laboratory biomarkers in comparison with a simplified clinical score for risk stratification of patients with acute pulmonary embolism. Chest. 2012;141:916-22.

25. Ozsu S, Ozlu T, Sentürk A, Uçar EY, Kırkıl G, Kadıoğlu EE, et al. Combination and comparison of two models in prognosis of pulmonary embolism: results from TUrkey Pulmonary Embolism Group (TUPEG) study. Thromb Res. 2014;133:1006-10.

26. Vanni S, Nazerian P, Pepe G, Baioni M, Risso M, Grifoni G, et al. Comparison of two prognostic models for acute pulmonary embolism: clinical versus right ventricular dysfunction-guided approach. J Thromb Haemost. 2011;9:1916-23.

27. Jiménez D, Kopecna D, Tapson V, Briese B, Schreiber D, Lobo JL, et al. Derivation and validation of multimarker prognostication for normotensive patients with acute symptomatic pulmonary embolism. Am J Respir Crit Care Med. 2014 Mar;189:718-26.

28. Konstantinides SV, Torbicki A, Agnelli G, Danchin N, Fitzmaurice D, Galiè N, et al. 2014 ESC guidelines on the diagnosis and management of acute pulmonary embolism: The Task Force for the Diagnosis and Management of Acute Pulmonary Embolism of the European Society of Cardiology (ESC) Endorsed by the European Respiratory Society (ERS). Eur Heart J. 2014;35:3033-69.

Cite this article as: Masotti L, Panigada G, Landini G, Pieralli F, Corradi F, Lenti S, et al. Comparison and combination of a hemodynamics/biomarkersbased model with simplified PESI score for prognostic stratification of acute pulmonary embolism: findings from a real world study. Int J Res Med Sci 2015;3:3230-7. 